\title{
Release of large polycyclic aromatic hydrocarbons and fullerenes by cosmic rays from interstellar dust
}

\section{Swift heavy ion irradiations of interstellar carbonaceous dust analogue}

\author{
T. Pino ${ }^{1}$, M. Chabot ${ }^{2}$, K. Béroff ${ }^{1}$, M. Godard ${ }^{3}$, F. Fernandez-Villoria ${ }^{1}$, K. C. Le ${ }^{1}$, L. Breuer $^{4}$, \\ M. Herder ${ }^{4}$, A. Wucher ${ }^{4}$, M. Bender ${ }^{5}$, D. Severin ${ }^{5}$, C. Trautmann ${ }^{5,6}$, and E. Dartois ${ }^{1,7}$ \\ ${ }^{1}$ Institut des Sciences Moléculaires d'Orsay (ISMO), CNRS, Univ. Paris Sud, Université Paris-Saclay, 91405 Orsay, France \\ e-mail: thomas.pino@u-psud.fr \\ 2 Institut de Physique Nucléaire d'Orsay (IPNO), IN2P3-CNRS, Univ. Paris Sud, Université Paris-Saclay, \\ 91405 Orsay, France \\ ${ }^{3}$ Centre de Sciences Nucléaires et de Sciences de la Matière (CSNSM), CNRS/IN2P3, Univ. Paris Sud, Université Paris-Saclay, 91405 \\ Orsay, France \\ ${ }^{4}$ Fakultät für Physik, Universität Duisburg-Essen, Duisburg, Germany \\ ${ }^{5}$ GSI Helmholtzzentrum für Schwerionenforschung, 64291 Darmstadt, Germany \\ ${ }^{6}$ Technische Universität Darmstadt, 64287 Darmstadt, Germany \\ ${ }^{7}$ Institut d'Astrophysique Spatiale (IAS), CNRS, Univ. Paris Sud, Université Paris-Saclay, 91405 Orsay, France \\ e-mail: emmanuel.dartois@u-psud.fr
}

Received 13 December 2018 / Accepted 3 February 2019

\begin{abstract}
Context. Top-down chemistry is believed to be responsible for the formation of the large molecular compounds such as the polycyclic aromatic hydrocarbon-like molecules and the fullerenes observed in the interstellar medium. The release of these large molecules from the parent grains remains an important issue to be investigated.

Aims. Cosmic rays irradiate the dust grains during their journey in the interstellar medium. In this study we probe to what extent electronic sputtering and/or desorption processes induced by high-energy ion projectiles contribute to the creation of the large molecular component in space.

Methods. Carbonaceous dust analogues were produced in an ethylene flame. The resulting soot nanoparticles generated under welldefined conditions were irradiated by swift heavy ions, and mass spectra of the ionic and neutral molecular fragments emitted shortly after the impact were monitored.

Results. Large molecular fragments were detected, including neutral and ionic polycyclic aromatic hydrocarbons containing up to several tens of carbon atoms, as well as ionic fullerenes. Although the absolute efficiencies were not obtained, these experiments provide a proof of principle of a top-down scenario involving interaction processes of interstellar dust with high-energy projectiles yielding large molecular compounds observed in space.
\end{abstract}

Key words. astrochemistry - cosmic rays - dust, extinction - ISM: molecules

\section{Introduction}

It is strongly suspected that large carbonaceous molecules carry the aromatic infrared bands (AIBs) observed in many lines of sight throughout the interstellar medium (ISM). The mid-IR range of wavelengths of the AIBs points to an out-of-equilibrium radiative relaxation mechanism triggered by the absorption of a single UV-starlight photon. The position of the bands and the emission mechanism led to the so-called polycyclic aromatic hydrocarbon (PAH) hypothesis and the presence of a molecular component in the form of polycyclic aromatic aliphatic mixed hydrocarbons is now well accepted (Sloan 2017; Jones et al. 2017; Yang et al. 2017; Pilleri et al. 2015; Sloan et al. 2014, 2007; Carpentier et al. 2012; Acke et al. 2010; Pino et al. 2008). The size of these large molecules ranges from a few tens to a few hundred atoms. Recently, neutral $\mathrm{C}_{60}$ and $\mathrm{C}_{70}$ (Sellgren et al. 2010; Cami et al. 2010) and possibly cationic $\mathrm{C}_{60}^{+}$(Berné et al. 2013) detected via their IR emission spectra confirmed the existence of such a large molecular component. The most recent assignment of five diffuse interstellar bands, observed in absorption in the near-infrared, to the vibronic bands of the first electronic transition in $\mathrm{C}_{60}^{+}$(Campbell et al. 2015, 2016; Walker et al. 2015) definitely proved that the ISM is enriched by such large carbonaceous molecules.

A gap in abundance between the largest molecule detected by rotational spectroscopy (11 atoms), with a column density decreasing drastically with size, and the fullerenes suggests that different formation mechanisms are at work. For the small interstellar molecules, homogeneous chemistry, gas-surface heterogeneous chemistry, and chemistry in ices are usually considered. However, these reactions cannot produce an amount of molecules as large as the fullerenes and the interstellar PAHs. Top-down mechanisms are therefore invoked and some models explored the possibility of formation of $\mathrm{C}_{60}$ from the large $\mathrm{PAH}$ component (Berne et al. 2015), relying on the photostability of these compounds in the ISM (Montillaud et al. 2013). However, 
these scenarios question the ability of the dust nanograins to release the PAHs and fullerene components. High-energy interaction processes are at the core of these scenarios where UV irradiation, irradiation with light or heavy ions and shocks may lead to the release of the molecules (Léger et al. 1985). To date, these processes have been studied to better constrain the enrichment of the ISM with small molecules (Alata et al. 2014, 2015; Dartois et al. 2017). The goal of the present work is to provide experimentally a proof of principle that cosmic rays (CRs), where energy deposition is dominated by the electronic energy loss, can lead to the emission of PAHs and fullerenes. Our results provide strong evidence in favour of a top-down scenario for the formation of free-flying large carbonaceous molecules in space.

The paper is organised as follows. A brief description of the experimental set-up is given. The results are then presented and analysed to provide the molecular species released upon swift heavy ions simulating CR by laboratory experiments. Finally, a discussion on the astrophysical implication and concluding remarks are reported.

\section{Experiment}

\subsection{Analogue production}

The analogues were produced using the nanograins set-up already described in previous publications (Pino et al. 2008; Carpentier et al. 2012; Gavilan et al. 2016, 2017). In brief, the soot samples were produced in a low-pressure ethylene flame burning at 40 mbar with a $\mathrm{C} / \mathrm{O}$ ratio of 1.05 . Ethylene and $\mathrm{O}_{2}$ flowed through the McKenna burner at a constant flow rate of $4 \mathrm{sl} \mathrm{min}^{-1}$ and the shielding $\mathrm{N}_{2}$ flow at $3 \mathrm{sl} \mathrm{min}^{-1}$. The height above the burner to extract the soot was set to $24 \mathrm{~mm}$. These conditions allow the production of the "young" soot. The sampling time was set to $10 \mathrm{~min}$ and the substrate was a plate of stainless steel (Omicron). Results from spectroscopy investigations of these soot particles in the IR-VUV range were reported recently (Gavilan et al. 2017). The study revealed that under these combustion conditions soot analogues of the circumstellar and interstellar polyaromatic dust are produced. The soot particles produced here were found to be good intermediates between classes $\mathrm{B}$ and $\mathrm{C}$ according to the AIB classification. A $\mathrm{C} / \mathrm{H}$ ratio of about 25 was found for this soot sample, representative of the polyaromatic dust grains in these objects (Gavilan et al. 2017).

\subsection{Ion irradiations}

The ion irradiations with swift heavy ions (SHI) were performed at the M1 branch of the UNILAC of GSI Helmholtzzentrum für Schwerionenforschung (Darmstadt, Germany). During the experiments, we used a ${ }^{48} \mathrm{Ca}^{n+}$ at $4.8 \mathrm{MeV} \mathrm{u}^{-1}$ swift heavy ion (SHI) beam (July 2016) with an ion current on the target of about $40 \mathrm{nA} \mathrm{cm}^{-2}$. Equilibrium charge states were obtained by inserting a carbon foil in the ${ }^{48} \mathrm{Ca}^{10+}$ beam delivered by the accelerator. The accelerator was operated at a frequency of $2 \mathrm{~Hz}$ and the length of the beam pulse was $5 \mathrm{~ms}$. The electronic stopping power, $19.91 \mathrm{MeV}\left(\mathrm{mg} \mathrm{cm}^{-2}\right)^{-1}$, was calculated using the SRIM-2013 code (Stopping and Range of Ions in Matter; Ziegler et al. 2010), with a $\mathrm{C} / \mathrm{H}$ of 25 and a density of $1.7 \mathrm{~g} \mathrm{~cm}^{-3}$. The range of the ions $(40 \mu \mathrm{m})$ is much larger than the thickness of the film (few $\mu \mathrm{m}$ ), ensuring homogeneous interaction with the target along the ion trajectory. The samples were exposed under a beam incidence of $45^{\circ}$.

\subsection{TOF mass spectrometry}

Mass spectra were monitored using a recently installed time-offlight mass spectrometry (TOFMS) unit operating in reflectron mode (Meinerzhagen et al. 2016). The optical axis of the TOFMS is set normal to the sample surface and the pulsed ion extraction is synchronised to the SHI pulsed beam. The MCP detector is floated at a post-acceleration potential of $-4 \mathrm{kV}$ with respect to the ground. In combination with the stage extraction potential of $1500 \mathrm{~V}$, this yields an ion impact energy of $5.3 \mathrm{keV}$ onto the MCP surface. We have not measured the detection efficiency as a function of the post-acceleration potential, but we woule like to note that the detection was performed in the so-called analogue mode, where the MCP output was performed by directly digitising the MCP output using a transient digitiser. In this operation mode, the measured signal will always increase with increasing post-acceleration voltage. All triggering events are described in detail in a previous publication (Meinerzhagen et al. 2016) where the complete chronograph is displayed. Secondary ions and secondary neutral mass spectra (SIMS and SNMS, respectively) are monitored within a single SHI pulse of $5 \mathrm{~ms}$ duration. In addition, each mass spectrum acquired during the UNILAC pulse is complemented by a blank spectrum taken during beam-off times between the ion pulses, but under otherwise identical experimental conditions. This is done by gating the acquisition with a pulse of the same length as the UNILAC pulses, but delayed by five times its width. For the SNMS, a photoionisation nanosecond pulsed laser operating at $157 \mathrm{~nm}$ ( $7.9 \mathrm{eV}$ photon energy) is fired few $\mathrm{mm}$ above the sample holder and parallel to it, typically $300 \mu \mathrm{s}$ after the beginning of the SHI pulse (and blank pulse). Since the TOFMS extraction is running at $1.2 \mathrm{kHz}$, it enables the accumulation of several SIMS spectra without laser (called repetitions), as compared to the SNMS spectra within the same acquisition cycle (event). Five hundred events were necessary to obtain a good SNMS signal-to-noise ratio $(\mathrm{S} / \mathrm{N})$. The mass resolution of the SNMS was found to be about 500 at mass $300 \mathrm{u}$ (corresponding to the mass of the coronene $\mathrm{C}_{24} \mathrm{H}_{12}$ molecule) and lower for the SIMS due to the large velocity dispersion of the ions and the better spatially defined ion cloud of the photoionised neutral molecules in the extraction zone of the TOFMS. The mass window was restricted to $0-1500 \mathrm{u}$ for this first set of experiments. With this experimental set-up, the secondary ion mass spectrum emitted shortly after the SHI impact was detected, as were the neutral species (Breuer et al. 2016). This unique design gives access to the detection of large neutral molecules and radicals released during the irradiation. The photoionisation wavelength for SNMS is particularly efficient and can perform the direct ionisation of species with an ionisation potential below $7.9 \mathrm{eV}$, i.e. conjugated molecules such as the PAHs.

\section{Results and analysis}

\subsection{Mass spectra}

The mass spectra are presented in Fig. 1 for the masses below $100 \mathrm{u}$, in Fig. 2 for the mass range between 100 and $600 \mathrm{u}$, and in Fig. 3 for the masses above $600 \mathrm{u}$. For the last, only the SIMS is shown because no species are detected in the SNMS. A data treatment was applied to normalise the intensities in counts per repetition. The normalised SIMS intensities were obtained by dividing the accumulated SIMS spectra by the total number of repetitions $\left(N_{\mathrm{TOT}}=N_{\text {events }} \times N_{\text {repet }}\right)$; the normalised SNMS intensities were obtained by dividing the accumulated "laser on" spectra by $N_{\text {events }}$ and subtracting the normalised SIMS spectra. 
T. Pino et al.: Release of large polycyclic aromatic hydrocarbons and fullerenes by cosmic rays from interstellar dust

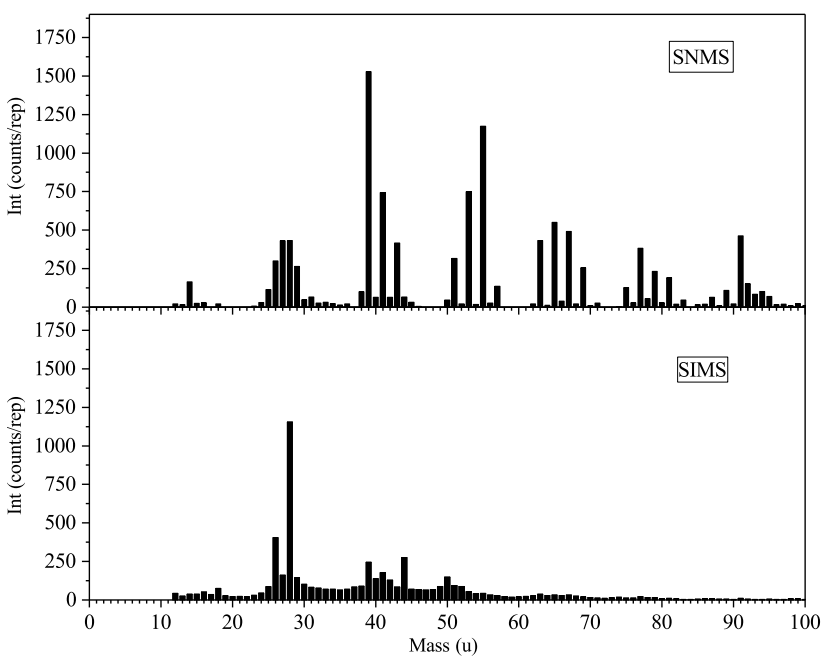

Fig. 1. SIMS and SNMS measured in the $0-100 \mathrm{u}$ (unified atomic mass unit) mass range.

Integration of the peaks around each mass unit was performed to plot the intensities directly. Because the soot target is composed mainly of carbon and hydrogen, a contribution of ${ }^{13} \mathrm{C}$ was taken into account. The natural abundance and all possible combinations were computed to perform the isotopic deconvolution. Because the structure of the SNMS can easily be identified with well-separated groups of peaks of species containing the same number of carbon atoms, the decomposition can be done as in Weilmunster et al. (1999) and every mass peak can be assigned to a chemical formula. For the SIMS data up to mass $600 \mathrm{u}$, the lower mass resolution and the presence of many peaks prevent the application of this simple protocol. We thus hypothesised that the species distribution were identical to those of the neutral for the 100-600 u mass range in order to perform the ${ }^{13} \mathrm{C}$ deconvolution of the SIMS. This was performed after background subtraction, as explained below.

\subsection{Secondary molecular ions}

Regarding the SIMS, few masses dominate the distribution: $\mathrm{C}_{2} \mathrm{H}_{2}, \mathrm{C}_{2} \mathrm{H}_{4}, \mathrm{C}_{3} \mathrm{H}_{3}, \mathrm{C}_{3} \mathrm{H}_{8}$, and $\mathrm{C}_{4} \mathrm{H}_{2}$. The broad distribution of masses at smaller intensities suggests contribution of intense dissociation of emitted molecular fragments. In principle, fragmentation of molecular ions during their flight along the field-free drift path of the mass spectrometer may also contribute additional peaks to the measured spectrum. While such contributions could in principle be identified by re-tuning the flight time focusing properties of the reflectron (see Boesl et al. 1982), such an analysis was not attempted here due to the limited beam time available. In addition to the mass resolved peaks, a broad unresolved background is detected which arises from fragmentation processes within the extraction region and/or incomplete flight time focusing of secondary ions extracted from regions outside the focal volume of the mass spectrometer (Meinerzhagen et al. 2016). For this background, no identification may be performed. Therefore, for the subsequent analysis, only the superimposed peaks revealing a regular pattern are discussed. Subtraction of the underlying continuum was performed in the SIMS data above $100 \mathrm{u}$ and only the remaining ion peaks were considered further (Figs. 2 and 3).

For the larger masses, the number of counts is by far smaller than those of the dominating peaks. However, the hydrogenation pattern remains with groups of peaks. These recall a typical distribution whose hydrogenation is found close to the most compact PAHs (such as coronene, ovalene, circumcoronene), although the $\mathrm{H} / \mathrm{C}$ ratios are slightly higher. Starting at mass $720 \mathrm{u}$, the SIMS data exhibit a regular distribution of peaks separated by 24 mass units. This is characteristic of a fullerene distribution (Kroto et al. 1985), the smallest one detected here being $\mathrm{C}_{60}^{+}$. Owing to the chosen mass window during this first set of experiments (below $1500 \mathrm{u}$ ), the complete distribution of the emitted fullerenes was not monitored and an instrumental cut-off at $\mathrm{C}_{124}$ is observed. It is noticeable that the mass spectrum exhibits the magic numbers of 60 and 70 carbon atoms and a broad distribution peaking above 100 carbon atoms.

\subsection{Secondary neutral molecules}

The SNMS in the low-mass range is dominated by species with an odd numbered hydrogenation. This is suggestive of a fragmentation pattern of larger species produced upon photoionisation because the ionisation potential of species containing a number of carbon atoms in this small mass range, even for the small aromatic molecules, is higher than the $7.9 \mathrm{eV}$ of the laser photon, and thus cannot be directly photoionised (Desgroux et al. 2013). Species released in metastable electronic states could also contribute via direct photoionisation. The number of counts/rep is found to be comparable to that of the ions (Fig. 1). However, the multiphoton ionisation process implies a much more efficient neutral emission of small species.

Above about $100 \mathrm{u}$, the distribution resembles typical PAH distributions that can be extracted from flames or observed by nanosecond laser desorption followed by laser ionisation (Apicella et al. 2009; Faccinetto 2009; Faccinetto et al. 2011). For instance, peaks at the masses of pyrene and coronene are found to dominate. Even numbers of carbon and even numbers of hydrogen are the structures that are measured to be the most abundant, as expected for a PAH distribution. Compared to the emitted ions, the number of counts/rep for neutral molecules is found to be much higher (Fig. 2). For instance the neutral to cation ratio at the mass of coronene $(300 \mathrm{u})$ is found to be about 50 .

The H/C distribution is plotted in Fig. 4, where the intensity of the peaks is traced as a function of the number of $\mathrm{C}$ and $\mathrm{H}$ in a 3D plot. The most intense species exhibit hydrogenation that closely follows that of the most compact PAHs, with one or two species dominating for a given number of $\mathrm{C}$, as illustrated by the inset in Fig. 2. The intensity distribution of species with lower $\mathrm{H}$ content probably arises either from fragmentation of the emitted PAHs after the SHI impact and/or is due to the photoionisation process. The intensity ratios were found not to depend strongly upon variation of the laser intensity, confirming a weak contribution of photodissociation. Therefore, the hydrogen distribution probably reflects the release process, and the dominating $2 \mathrm{H}-\mathrm{loss}$ channel at the mass of coronene (see inset in Fig. 2) helps to constrain the effective temperature of emission to about $2200 \mathrm{~K}$ (Chen et al. 2015).

\section{Discussion}

\subsection{PAHs and fullerenes released by cosmic radiation}

By irradiating soot samples with swift heavy ions, we identified neutral and cationic PAHs as well as cationic fullerenes. Using the geometrical configuration of the set-up, the photoionisation cross section at $7.9 \mathrm{eV}$ of coronene of about 10 Mbarn (Johansson et al. 2017; Verstraete et al. 1990), and a $20 \mathrm{~mJ} \mathrm{~cm}^{-2}$ pulse $\mathrm{s}^{-1}$ energy density, a rough estimation of the 


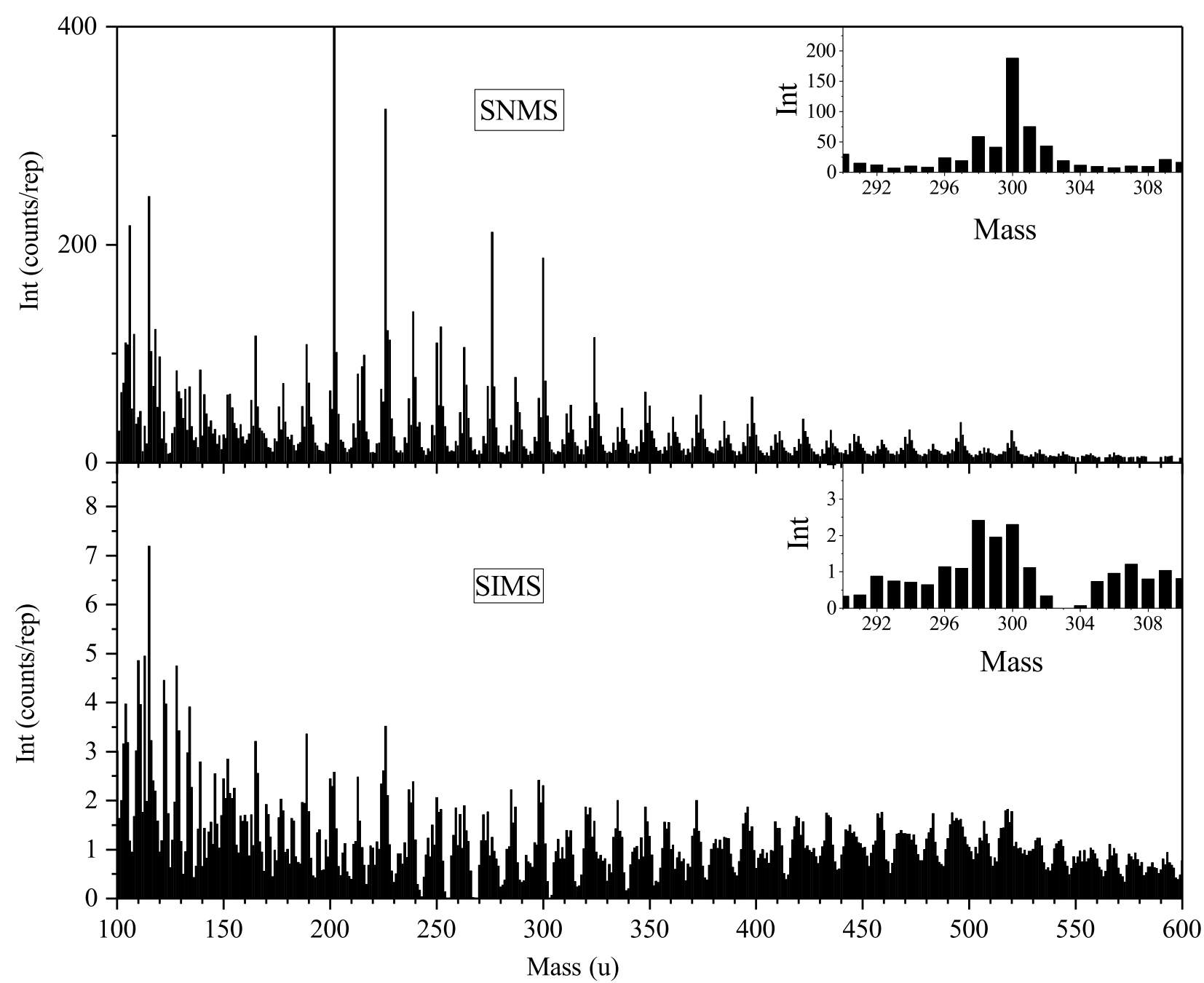

Fig. 2. SIMS and SNMS measured in the 100-600 u mass range. Insets are zoom-ins around mass 300 corresponding to the mass of coronene $\mathrm{C}_{24} \mathrm{H}_{12}$.

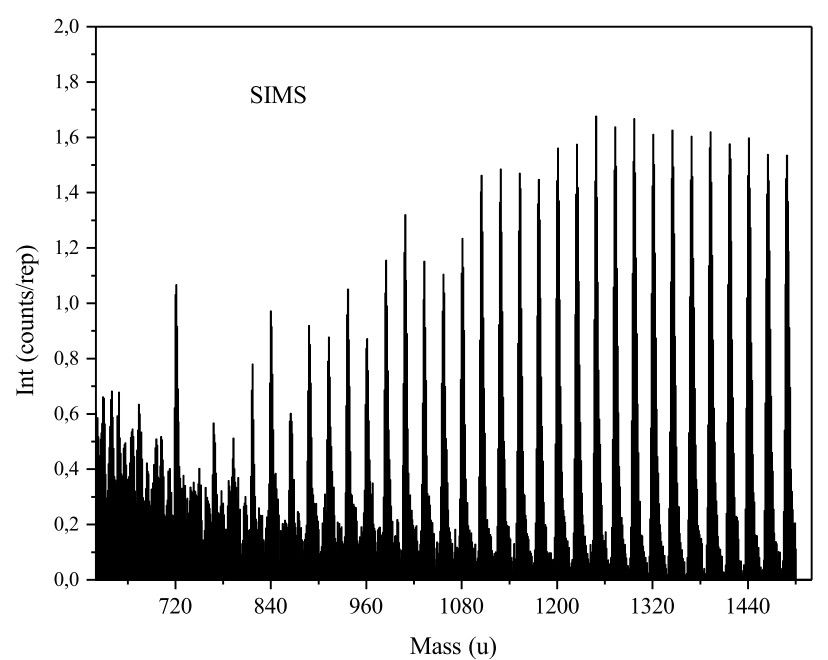

Fig. 3. SIMS measured in the mass range above $620 \mathrm{u}$. Note that a linear background was removed.

photoionisation efficiency gives 0.1 . Using this efficiency and the measured neutral to cation ratio for coronene, the ratio of emitted cation to neutral is deduced to be about $2 \times 10^{-3}$. This

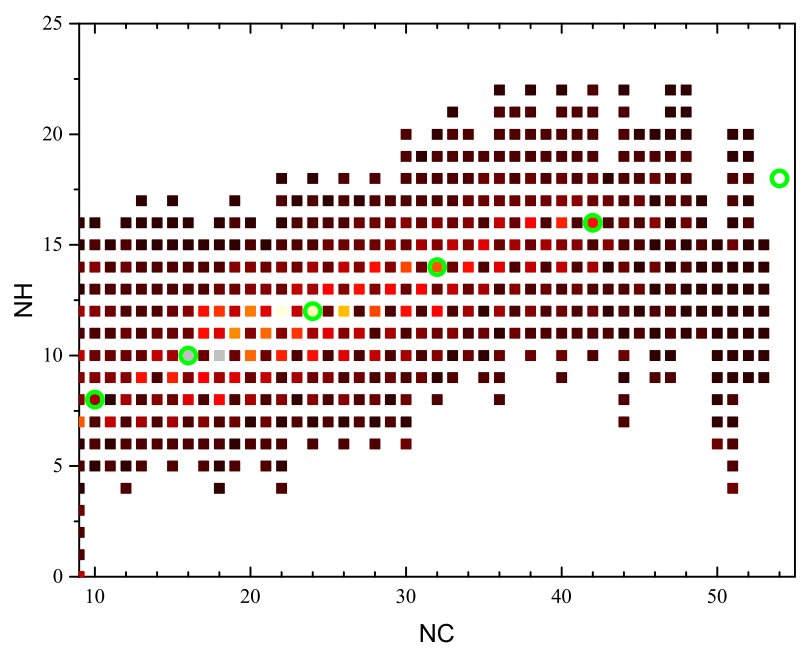

Fig. 4. Number of carbon NC and hydrogen NH in the neutral PAH mass range. The colours scale with the intensity of the peaks increasing from black to red and finally yellow. The open green circles mark the $\mathrm{H}$ and $\mathrm{C}$ composition of compact PAHs up to circumcoronene. Lower $\mathrm{H}$ content probably arises from emitted partially dehydrogenated fragments, while higher probably traces less compact structures. 
value is comparable to the emission of secondary cations and neutrals measured for a coronene film (Breuer et al. 2016). PAHs could be present at the surface of the soot, although in low concentration. Because the neutral PAH distribution is very close to that measured from flames, for example using laser induced desorption in the nanosecond regime (Faccinetto et al. 2011, 2015; Alfe et al. 2008), the SNMS may be dominated by these adsorbed PAHs.

A striking point is the detection of cationic fullerenes with no evidence of neutral ones. It raises questions about the emission/production mechanism of these species. Detection of ionic fullerenes upon $\mathrm{MeV}$ ion irradiations has already been reported (Brinkmalm et al. 1992, 1993; Bitensky et al. 1993, 1994; Demirev et al. 1994; Akhtar et al. 1997; Qayyum et al. 1998). Influence of the nature of the target was shown with cluster growth starting from $\mathrm{C}_{60}$ when it was already present in the material (Bitensky et al. 1994; Demirev et al. 1994; Papaleo et al. 1996). Our SIMS shows a fullerene distribution starting at $\mathrm{C}_{60}$, suggesting that the present soot material may already contain fullerenic structures. According to its spectroscopy (Gavilan et al. 2017), this soot is made of $\mathrm{sp}^{2}$ carbons with defects causing curvature and tortuosity. In Carpentier et al. (2012), the soot was also consistently shown to possess curved structures. The initial structure of the soot particle may thus favour the formation and emission of fullerenes, although it does not contain such fullerenic structures. It is also noteworthy that in the present experiment, the electronic stopping power was 19.91 $\mathrm{MeV}\left(\mathrm{mg} \mathrm{cm}^{-2}\right)^{-1}$, just above the threshold for fullerene formation and emission upon SHI impact that was predicted to be in the range $10-20 \mathrm{MeV}\left(\mathrm{mg} \mathrm{cm}^{-2}\right)^{-1}$ (Bitensky et al. 1993). In addition, their release was demonstrated to originate from the core region of the tracks (Brinkmalm et al. 1993; Bitensky et al. 1993, 1994; Demirev et al. 1994; Papaleo et al. 1996). Moreover, for an indium and silver targets, the ionisation probability were shown to depend on the projectile impact angle, with ionisation being enhanced for large angles of beam incidence (Mai et al. 2013). Therefore, the detection of fullerenic neutral species is probably unfavoured compared to cationic species; incident ions impacting at $45^{\circ}$ with respect to the TOF axis have obviously a larger ion yield and favours the emission of the neutral fullerenes along the ion track and not normal to the surface. This also implies that that they may escape the post-photoionisation zone of the TOFMS. In addition, formation of ionised metastable fullerene fragments may actually be preferred as a result of the competition between thermionic electron emission (Campbell et al. 1991, 1992; Walder et al. 1993) and dissociation through $\mathrm{C}_{2}$ emission during relaxation of the emitted hot fullerenes from the ion track. In the ISM, the formation of the fullerenes may be triggered by the cosmic rays and subsequently emitted from the highly excited core region of the ion tracks.

\subsection{Plausible top-down scenario?}

The astrophysical relevance of the electronic sputtering induced by CRs on interstellar carbonaceous dust to the production of large molecular species in the ISM relies on the efficiency of the process. While it is not yet measurable in the laboratory, astrophysical constraints can be set to explore its likelihood. Previous sputtering yield measurements can be used to infer the $\mathrm{C}$ sputtering rate in an interstellar Galactic cosmic ray exposed environment. The sputtering rate for carbon $R_{\mathrm{C}}$ (given in $\mathrm{C}$ atom $\mathrm{cm}^{-2} \mathrm{~s}^{-1}$ ) $R_{\mathrm{C}}^{\mathrm{GCR}}$ can be evaluated by integrating the product of the yield with the cosmic ray flux over their energy and abundance distribution following

$R_{\mathrm{C}}^{\mathrm{GCR}}\left[\mathrm{C}\right.$ atom $\left.\mathrm{cm}^{-2} \mathrm{~s}^{-1}\right]=4 \pi \sum_{\mathrm{Z}} \int_{E_{\min }}^{\infty} Y_{\mathrm{s}}^{\mathrm{CR}}(E, Z) \frac{\mathrm{d} N}{\mathrm{~d} E}(E, Z) \mathrm{d} E$,

where $\frac{\mathrm{d} N}{\mathrm{~d} E}(E, Z)\left[\right.$ particles $\left.\mathrm{cm}^{-2} \mathrm{~s}^{-1} s r^{-1}\left(M e V u^{-1}\right)^{-1}\right]$ is the differential flux of the cosmic ray element of atomic number $Z$ and energy per nucleon $E$, with a cut-off in energy $E_{\min }$ set at $100 \mathrm{eV}$. Moving the cut-off from $10 \mathrm{eV}$ to $1 \mathrm{keV}$ does not change the results significantly. The sputtering yield $Y_{\mathrm{s}}^{\mathrm{CR}}(E, Z)$ is evaluated by combining $Y_{\mathrm{s}}(\mathrm{Se})$ established experimentally and the calculated electronic stopping cross section $\operatorname{Se}(E, Z)$ using the SRIM code (Ziegler et al. 2010) as a function of atomic number $Z$ and energy per nucleon $E$. For the differential flux, we adopt the functional form given by Webber \& Yushak (1983), also described in Shen et al. (2004),

$$
\frac{\mathrm{d} N}{\mathrm{~d} E}(E, Z)=f(Z) \frac{C E_{0}^{0.3}}{\left(E+E_{0}\right)^{3}},
$$

where $\mathrm{C}$ is a normalisation constant and $E_{0}$ a form parameter allowing us to account for the low-energy cosmic ray distribution.

To perform a calculation on the rate in interstellar space, we adopt the sputtering yield measurements given by Ghosh et al. (2004) for amorphous carbon, measured at $100 \mathrm{MeV}\left(\mathrm{mg} \mathrm{cm}^{-2}\right)^{-1}$, of $1.8 \times 10^{4} \mathrm{C}$ atoms/ion and assuming a quadratic dependency of the sputtering yield with the stopping power in the electronic regime. The SRIM stopping power cross sections for the different interstellar ions are calculated with a density of $1.7 \mathrm{~g} \mathrm{~cm}^{-3}$, corresponding to typical soot value. The corresponding rate gives $1.24 \mathrm{C}$ atom $\mathrm{cm}^{-2} \mathrm{~s}^{-1} / 10^{16} \zeta$, where $\zeta$ $\left(\mathrm{s}^{-1}\right)$ is the cosmic ray ionisation rate. The Jones et al. (2017) model predicts a carbonaceous nanoparticle surface density of about $1.7 \times 10^{-20} \mathrm{~cm}^{2}$ per hydrogen atom. The overall production rate is therefore $2.1 \mathrm{C}$ atom $10^{-36} / \zeta \mathrm{H}^{-1} \mathrm{~s}^{-1}$.

The rate of production of a species corresponds therefore to this rate multiplied by the fraction $\eta$ of a species produced in the sputtering process. The $\mathrm{C}$ fraction locked in the $C_{60}^{+}$ fullerene from diffuse absorption bands in the visible is about $0.05-0.1 \%$ of the carbon abundance, i.e. $\sim 10^{-7} / \mathrm{H}$. Adopting an ionisation rate of $\zeta=10^{-16} \mathrm{~s}^{-1}$, waiting $100 \mathrm{My}$ would require $\eta \sim 1.5 \times 10^{-3}$ to explain the observed abundance. This efficiency is that of the sputtered carbon finally locked into the interstellar $C_{60}^{+}$fullerenes. This value cannot rule out the contribution of electronic sputtering and calls for quantitative studies.

\section{Concluding remarks}

Emitted molecular fragments from a laboratory analogue of carbonaceous interstellar dust irradiated by swift heavy ion simulating cosmic ray effects were measured. Large PAHs have been detected with the neutral population dominating over the cations. This experimental results highlights the importance of the post-photoionisation facility to monitor the release of neutral molecules. The distribution of the neutral PAHs suggests desorption of rather intact adsorbed PAHs even with sizes as high as PAHs containing up to 50 carbon atoms. Cationic fullerenes up to 120 carbon atoms were observed with a broad mass distribution. Geometrical constraints probably prevented the detection of neutral fullerenes, but this has still to be proven. These experiments provide a proof of principle of a top-down 
scenario for the production of large carbonaceous molecules in the ISM involving cosmic radiation of interstellar dust and electronic sputtering. Efficiencies have to be measured to quantify the effective contribution of this energetic process.

Acknowledgements. Financial support from the CNRS/INSU French nationa program on Physics and Chemistry of the Interstellar Medium is acknowledge. This work has been supported by the European Union's Horizon 2020 research and innovation program and grant agreement number 6544002.

\section{References}

Acke, B., Bouwman, J., Juhasz, A., et al. 2010, ApJ, 718, 558

Akhtar, M., Ahmad, B., \& Ahmad, S. 1997, Phys. Lett. A, 234, 367

Alata, I., Cruz-Diaz, G. A., Munoz Caro, G. M., \& Dartois, E. 2014, A\&A, 569, A119

Alata, I., Jallat, A., Gavilan, L., et al. 2015, A\&A, 584, A123

Alfe, M., Apicella, B., Tregrossi, A., \& Ciajolo, A. 2008, Carbon, 46, 2059

Apicella, B., Wang, X., Armenante, M., Bruno, A., \& Spinelli, N. 2009, in Proceedings of the 32nd Meeting on Combustion, Naples, Italy, I-12

Berné, O., Mulas, G., \& Joblin, C. 2013, A\&A, 550, L4

Berne, O., Montillaud, J., \& Joblin, C. 2015, A\&A, 577, A133

Bitensky, I., Demirev, P., \& Sundqvist, B. 1993, Nucl. Instrum. Methods Phys. Res. B, 82, 356

Bitensky, I., Brinkmalm, G., Demirev, P., et al. 1994, Int. J. Mass Spectrom. Ion Process., 138, 159

Boesl, U., Neusser, H., Weinkauf, R., \& Schlag, E. 1982, J. Phys. Chem., 86, 4857

Breuer, L., Meinerzhagen, F., Herder, M., et al. 2016, J. Vac. Sci. Technol. B, 34, $03 \mathrm{H} 130$

Brinkmalm, G., Barofsky, D., Demirev, P., et al. 1992, Chem. Phys. Lett., 191, 345

Brinkmalm, G., Demirev, P., Fenyo, D., et al. 1993, Phys. Rev. B, 47, 7560

Cami, J., Bernard-Salas, J., Peeters, E., \& Malek, S. 2010, Science, 329, 1180

Campbell, E., Ulmer, G., \& Hertel, I. 1991, Phys. Rev. Lett., 67, 1986

Campbell, E., Ulmer, G., \& Hertel, I. 1992, Z. Phys. D. Atom. Mol. Cluster., 24,81

Campbell, E., Holz, M., Gerlich, D., \& Maier, J. 2015, Nature, 523, 322

Campbell, E., Holz, M., Maier, J., et al. 2016, ApJ, 822, 17

Carpentier, Y., Féraud, G., Dartois, E., et al. 2012, A\&A, 548, A40

Chen, T., Gatchell, M., Stockett, M. H., et al. 2015, J. Chem. Phys., 142, 144305

Dartois, E., Chabot, M., Pino, T., et al. 2017, A\&A, 599, A130
Demirev, P., Brinkmalm, G., Eriksson, J., et al. 1994, Carbon, 32, 809

Desgroux, P., Mercier, X., \& Thomson, K. 2013, Proc. Combust. Inst., 34, 1713

Faccinetto, A. 2009, PhD Thesis, Université Lille 1, France

Faccinetto, A., Desgroux, P., Ziskind, M., Therssen, E., \& Focsa, C. 2011, Combust. Flame, 158, 227

Faccinetto, A., Focsa, C., Desgroux, P., \& Ziskind, M. 2015, Environ. Sci. Technol., 49, 10510

Gavilan, L., Alata, I., Le, K. C., et al. 2016, A\&A, 586, A106

Gavilan, L., Le, K. C., Pino, T., et al. 2017, A\&A, 607, A73

Ghosh, S., Avasthi, D. K., Tripathi, A., et al. 2004, Nucl. Instrum. Methods Phys. Res. B, 219, 973

Johansson, K. O., Dillstrom, T., Elvati, P., et al. 2017, Proc. Combust. Inst., 36, 799

Jones, A. P., Kohler, M., Ysard, N., Bocchio, M., \& Verstraete, L. 2017, A\&A, 602, A46

Kroto, H., Heath, J., O’Brien, S., Curl, R., \& Smalley, R. 1985, Nature, 318, 162 Léger, A., Jura, M., \& Omont, A. 1985, A\&A, 144, 147

Mai, M., Weidtmann, B., Marpe, M., \& Wucher, A. 2013, Nucl. Instrum. Methods Phys. Res. B, 317, 130

Meinerzhagen, F., Breuer, L., Bukowska, H., et al. 2016, Rev. Sci. Instrum. Inst., 87

Montillaud, J., Joblin, C., \& Toublanc, D. 2013, A\&A, 552, A15

Papaleo, R., Demirev, P., Eriksson, J., Hakansson, P., \& Sundqvist, B. 1996, Int. J. Mass Spectrom. Ion Process., 152, 193

Pilleri, P., Joblin, C., Boulanger, F., \& Onaka, T. 2015, A\&A, 577, A16

Pino, T., Dartois, E., Cao, A.-T., et al. 2008, A\&A, 490, 665

Qayyum, A., Ahmad, B., Akhtar, M., \& Ahmad, S. 1998, Eur. Phys. J. D, 3, 267

Sellgren, K., Werner, M., Ingalls, J., et al. 2010, ApJ, 722, L54

Shen, C. J., Greenberg, J. M., Schutte, W. A., \& van Dishoeck E. F. 2004, A\&A, 415,203

Sloan, G. C. 2017, Planet. Space Sci., 149, 32

Sloan, G., Jura, M., Duley, W., et al. 2007, ApJ, 664, 1144

Sloan, G. C., Lagadec, E., Zijlstra, A. A., et al. 2014, ApJ, 791

Verstraete, L., Léger, A., DHendecourt, L., Dutuit, O., \& Defourneau, D. 1990, A\&A, 237, 436

Walder, G., Kennedy, K., \& Echt, O. 1993, Z. Phys. D. Atom. Mol. Cluster., 26, S288

Walker, G., Bohlender, D., Maier, J., \& Campbell, E. 2015, ApJ, 812, L8

Webber, W. R., \& Yushak, S. M. 1983, ApJ, 275, 391

Weilmunster, P., Keller, A., \& Homann, K. 1999, Combust. Flame, 116, 62

Yang, X. J., Glaser, R., Li, A., \& Zhong, J. X. 2017, New Astron. Rev., 77, 1

Ziegler, J. F., Ziegler, M. D., \& Biersack, J. P. 2010, Nucl. Instrum. Methods Phys. Res. B, 268, 1818 